\title{
Gütesiegel für die Darmkrebsvorsorge
}

\author{
Die Mortalität infolge kolorektaler Karzinome ist in Ländern mit entsprechenden Vorsorge- \\ untersuchungen gesunken. Deutschland liegt erfreulicherweise in der Spitzengruppe.
}

— Mit den Daten der Weltgesundheitsorganisation wurde retrospektiv die alters- und geschlechtsspezifische Mortalität infolge kolorektaler Karzinome in 34 europäischen Ländern zwischen 1988 und 2011 analysiert. In 27 EU-Ländern ist die Mortalität bei Männern im Mittel um $13 \%$ gesunken.

Es bestehen aber erhebliche Unterschiede in den einzelnen Ländern. Während die Mortalität in Nord- und Westeuropa deutlich zurückging, verzeichneten viele südliche, zentrale und östliche Länder sogar einen Anstieg. Am schlechtesten schnitten Rumänien mit $+102 \%$ und Griechenland mit $+43,5 \%$ ab. Eine Ausnahme bildet Tschechien. Aber auch unter den wirtschaftsstarken Ländern gibt es Unterschiede: Die Niederlande verzeichneten einen Rückgang von $11,2 \%$, Schweden lag bei $14,9 \%$. In Österreich, der Schweiz, Deutschland und England waren es hingegen über 35\%. In den 34 Ländern nahm die Sterblichkeit bei Männer median um $6 \% \mathrm{zu}$, bei Frauen ging sie um 14,7\% zurück. Die USA kamen insgesamt auf einen Rückgang um $39,8 \%$.

- Ouakrim D A, Pizot C, Boniol M et al. Trends in colorectal cancer mortality in Europe: retrospective analysis of the WHO mortality database. BMJ. 2015;351:h4970

\section{KOMMENTAR}

Seit etwa 1970 hat die Mortalität infolge kolorektaler Karzinome in vielen Ländern abgenommen. Dieser Trend setzt sich bis heute fort. Viele Faktoren haben dazu beigetragen: Demografie, Lebensstiländerungen, Krank- heitsbewusstsein, Vorsorgeuntersuchungen wie die Koloskopie und bessere Behandlungsmöglichkeiten wie die Polypektomie. Es ist zwar mittlerweile in allen europäischen Ländern möglich, auf Eigeninitiative oder nach Arztempfehlung an Vorsorgeuntersuchungen teilzunehmen.

Nun zeigt sich also, dass die Trends sehr unterschiedlich sind - nicht nur in wirtschaftlich schwachen, sondern auch in florierenden Staaten. Hinweise auf einen unterschiedlichen Umgang mit Vorsorgeaktivitäten gibt es schon lange. Beispielsweise ist der Zugang zu endoskopischen Untersuchungen in den Niederlanden deutlich schwieriger als in Frankreich, Österreich oder Deutschland. Dies könnte zu den schlechteren Ergebnissen hinsichtlich der Mortalität beitragen.

Prof. Dr. med. H. Holzgreve

\section{Morbus Crohn beim Zahnarzt}

Ein 13-jähriger Junge hatte seit neun Monaten eine einseitige Gesichtsschwellung und Schmerzen im Bereich der Mundhöhle. Auf Nachfragen berichtete er auch von gelegent-

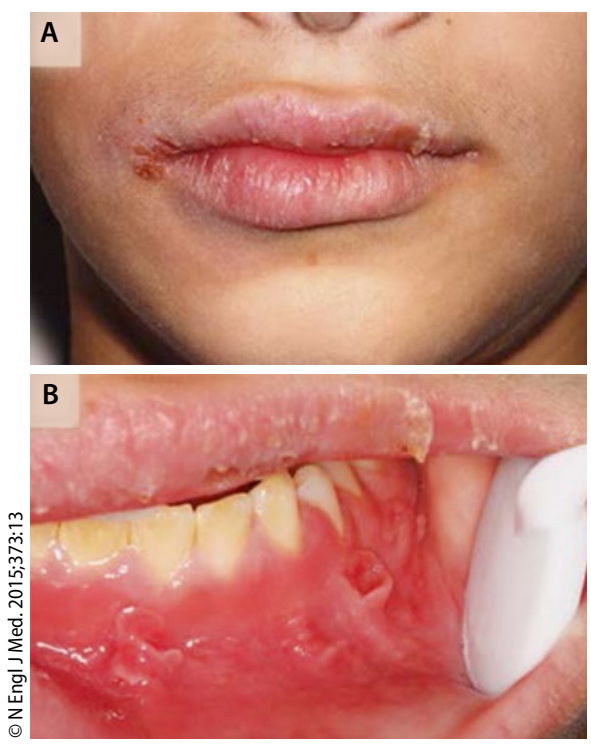

lichen dünnen Stühlen ohne Blutbeimengung. Es fanden sich eine asymmetrische Gesichtsschwellung, geschwollene Lippen mit Mundwinkelrhagaden (Abb. A) und ein leichtgeröte-

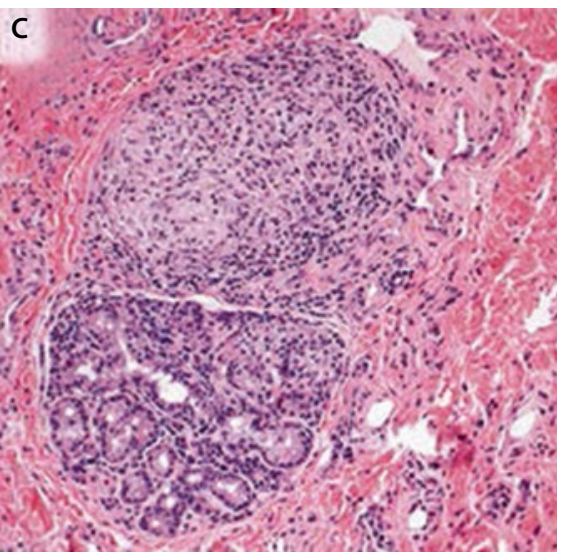

A: Geschwollene Lippen, Mundwinkelrhagaden; B: Teilweise ulzerierte Gewebelappen am Zahnfleisch; C: Granulationsgewebe im histologischen Schnittbild. tes Zahnfleisch mit Epulis-artigen, teilweise ulzerierten Gewebelappen (Abb. B). Histologisch fand sich Granulationsgewebe, das mit einem Morbus Crohn vereinbar war (Abb. C). Daraufhin wurde der Junge zum pädiatrischen Gastroenterologen überwiesen, wo man einen Druckschmerz im rechten unteren Quadranten und in der Periumbilikalregion, eine Analfissur und Marisken feststellte. Biopsien aus der Kolonschleimhaut ergaben eine chronische aktive Kolitis mit einer Verteilung vorwiegend im Zökum und im Colon ascendens. Somit war die Diagnose eines Morbus Crohn bestätigt.

Der Junge wurde mit Mesalazin und Prednison behandelt. Unter Erhaltungstherapie mit Mercaptopurin bildeten sich die Veränderungen in der Mundhöhle im Lauf eines Jahres zurück. Bei einer Nachfolgeuntersuchung zwei Jahre später war der Junge nach wie vor asymptomatisch.

Prof. Dr. med. H. S. FüeßI

- Aguirre A, Nugent CA (aguirr@buffalo.edu).Oral manifestation of Crohn's disease. N Engl J Med. 2015;373:13 